\title{
Life-threatening hyperkalaemia due to trimethoprim in a patient treated for Pneumocystis jirovecii pneumonia
}

\author{
N du Plooy, ${ }^{1} \mathrm{MB}$ ChB; B Rayner, ${ }^{2} \mathrm{MB}$ ChB, MMed, FCP, PhD \\ ${ }^{1}$ Department of Medicine, Faculty of Health Sciences, University of Cape Town and Groote Schuur Hospital, Cape Town, South Africa \\ ${ }^{2}$ Head, Nephrology and Hypertension, Department of Medicine, Faculty of Health Sciences, University of Cape Town, South Africa
}

Corresponding author: $N$ du Plooy (nielduplooy@gmail.com)

\begin{abstract}
Hyperkalaemia is a potentially life-threatening condition frequently encountered in hospitalised patients. Among the many causes of hyperkalaemia, drugs have often been implicated. In the South African context, with the high burden of HIV, there is an increased incidence of opportunistic infections such as Pneumocystis jirovecii pneumonia (PJP), and consequently many patients receive high doses of trimethoprim-sulfamethoxazole. A lesser-known side-effect of the trimethoprim component of this combination antibiotic is hyperkalaemia. We report a case in which life-threatening hyperkalaemia developed after institution of high-dose co-trimoxazole for PJP.
\end{abstract}

S Afr Med J 2019;109(2):89-90. DOI:10.7196/SAMJ.2019.v109i2.13660

Hyperkalaemia is a potentially life-threatening condition frequently encountered in hospitalised patients. ${ }^{[1,2]}$ It is associated with increased inpatient and all-cause mortality. ${ }^{[3]}$ Various causes for hyperkalaemia exist, which can be identified with relative ease. These include increased intake of potassium in the form of supplementation or iatrogenic administration, increased intracellular potassium release (e.g. rhabdomyolysis or haemolysis), and decreased excretion (e.g. in renal failure). Medication is arguably the most common cause of hyperkalaemia and can be implicated in $75 \%$ of cases. The most common medications include angiotensin-converting enzyme inhibitors (ACE-Is), angiotensin receptor blockers (ARBs), digoxin, spironolactone and non-steroidal anti-inflammatories. ${ }^{[1,4]}$

We have a very high burden of HIV in South Africa (SA). As a result there is an increased incidence of opportunistic infections such as Pneumocystis jirovecii pneumonia (PJP) and both acute and chronic kidney disease due to HIV-associated nephropathy. ${ }^{[5]}$ Patients with PJP require high doses of trimethoprim-sulfamethoxazole, which may cause life-threatening complications in the context of kidney disease.

We report one such case, in which life-threatening hyperkalaemia developed after institution of high-dose trimethoprim-sulfamethoxazole (co-trimoxazole) for PJP.

\section{Case report}

A 50-year-old woman with refractory hyperkalaemia was referred to the Renal Unit at Groote Schuur Hospital, Cape Town, SA. She was a known epileptic and was HIV-positive, with a history of poor adherence to treatment. She had defaulted on her antiretroviral medication for the past 2 years. Her CD4+ T-lymphocyte count was 6 cells $/ \mu \mathrm{L}$ and her HIV viral load 456362 copies $/ \mathrm{mL}$.

The patient was admitted to hospital with breakthrough seizures. After her seizures had been controlled, it was noted that she was dyspnoeic and hypoxic on room air, and it was reported that she had had respiratory symptoms during the preceding week. Her blood pressure on admission was $121 / 58 \mathrm{mmHg}$, her pulse rate 79 beats/ min and her temperature $35.7^{\circ} \mathrm{C}$. Her arterial oxygen saturation was $65 \%$ on room air and increased to $97 \%$ on non-rebreather mask oxygen. Her kidney function was normal (serum creatinine $77 \mu \mathrm{mol} / \mathrm{L}$, urea $7.9 \mathrm{mmol} / \mathrm{L}$, sodium $134 \mathrm{mmol} / \mathrm{L}$ and potassium $4.8 \mathrm{mmol} / \mathrm{L}$ ). A chest radiograph revealed bilateral ground-glass opacifications. Based on the radiological findings, hypoxaemia and severe immune compromise, a diagnosis of PJP was made. Trimethoprim-sulfamethoxazole $1600 \mathrm{mg} / 320 \mathrm{mg}$ 6-hourly and prednisone $40 \mathrm{mg} / \mathrm{d}$ was started. The possibility of aspiration pneumonia was a concern, and ceftriaxone $1 \mathrm{~g} / \mathrm{d}$ and azithromycin $500 \mathrm{mg} / \mathrm{d}$ were therefore also administered.

The patient responded well within the first week of her admission. By day 8 her creatinine had almost doubled, and by the 10th day her blood results were as follows: creatinine $309 \mu \mathrm{mol} / \mathrm{L}$, urea $38.4 \mathrm{mmol} / \mathrm{L}$, sodium $136 \mathrm{mmol} / \mathrm{L}$ and potassium $8.8 \mathrm{mmol} / \mathrm{L}$. Clinically she was dehydrated. After administration of intravenous saline, she passed approximately $600 \mathrm{~mL}$ of clear urine. She received calcium gluconate, and attempts to lower her serum potassium with intravenous dextrose and insulin were futile. Oral sodium polystyrene sulfonate was also administered without success, and she had emergency haemodialysis to correct her potassium.

In the interim, the trimethoprim-sulfamethoxazole was discontinued and clindamycin and primaquine were started once the results of glucose-6-phosphate dehydrogenase (G6PD) assay was known. G6PD deficiency is an X-linked disorder that renders erythrocytes vulnerable to oxidative stress and haemolysis. Primaquine, like all 8-aminoquinoline compounds, can result in severe haemolysis in individuals with G6PD deficiency, and screening for this enzyme defect is recommended before these medications are commenced. ${ }^{[6]}$ During the following days, the patient's condition improved rapidly. By 7 days after stopping trimethoprim-sulfamethoxazole, her renal function and potassium had returned to normal (creatinine $46 \mu \mathrm{mol} / \mathrm{L}$, urea $7.3 \mathrm{mmol} / \mathrm{L}$, sodium $136 \mathrm{mmol} / \mathrm{L}$ and potassium $3.8 \mathrm{mmol} / \mathrm{L}$ ).

\section{Discussion}

The combination antibiotic trimethoprim-sulfamethoxazole can affect kidney function in various ways. Firstly (and the most familiar renal side-effect associated with sulphonamide antibiotics) 
is acute interstitial nephritis. ${ }^{[7]}$ Secondly, in rare cases sulphonamides can cause crystal nephropathy, especially in an acidic, low-flow environment. Characteristic birefringent crystals in a 'shock-ofwheat' arrangement are observed on urine microscopy. ${ }^{[7]}$ Thirdly, trimethoprim interferes with creatinine secretion in the proximal convoluted tubule. This results in increased serum creatinine without affecting the glomerular filtration rate (GFR). This effect should therefore not be considered to be due to acute kidney injury. ${ }^{[7]}$ Lastly, trimethoprim results in natriuresis and antikaliuresis. Trimethoprim blocks the epithelial sodium channels (ENaCs) in the distal convoluted tubules and collecting ducts. This effect is similar to the effect seen with the potassium-sparing diuretic amiloride. Indeed, trimethoprim and amiloride are structurally similar. ${ }^{[7]}$

In our case, the sequence of events was probably development of prerenal failure due to the natriuretic effect of trimethoprim, resulting in further accumulation of trimethoprim and increasing the risk of hyperkalaemia.

A 2015 retrospective study described two significant risk factors for the development of hyperkalaemia with trimethoprim use, namely pre-existing renal impairment (estimated GFR (eGFR) $<60 \mathrm{~mL} / \mathrm{min} / 1.73 \mathrm{~m}^{2}$ ) and concomitant use of an ACE-I/ARB. The odds ratios for hyperkalaemia with low-dose trimethoprimsulfamethoxazole in these conditions have been reported as 4.62 and 3.96, respectively. ${ }^{[8]}$ Advanced age is also a recognised risk factor, as is the use of higher doses of trimethoprim. ${ }^{[9]}$ A 2014 review of nearly 40000 patients aged $>66$ years on an ACE-I or ARB who had an unexplained sudden death within 14 days of receiving outpatient antibiotic prescriptions found an odds ratio for sudden death of 1.54 in patients who received trimethoprim-sulfamethoxazole compared with those receiving amoxicillin. This translates to $\sim 3$ deaths per 1000 trimethoprim-sulfamethoxazole prescriptions within 14 days of receiving trimethoprim-sulfamethoxazole. ${ }^{[9]}$

Certain measures have been proposed to minimise the risk of hyperkalaemia. Firstly, all patients receiving trimethoprimsulfamethoxazole should have their doses adjusted according to their current renal function. The current recommendation is to give usual doses every 18 - 24 hours if the eGFR is $30-60 \mathrm{~mL} / \mathrm{min}$, and a half dose every $12-18$ hours if the GFR is $15-30 \mathrm{~mL} / \mathrm{min}$. Furthermore, it is recommended to avoid the drug when the GFR is $<15 \mathrm{~mL} /$ min. ${ }^{[10]}$ The second strategy is to increase the sodium load to the distal nephron with intravenous saline. This increases the substrate for the $\mathrm{ENaCs}$ and increases flow rates, thereby minimising the effect of trimethoprim. Thirdly, it has been proposed that urinary alkalinisation decreases the amount of protonated form of the drug, also decreasing its efficacy. ${ }^{[8]}$

Hyperkalaemia should be addressed appropriately according to the serum potassium level. When it is established that hyperkalaemia is secondary to trimethoprim, the offending drug (and others that might exacerbate the situation) must be discontinued with immediate effect. Perazella ${ }^{[9]}$ suggested that it may be reinitiated, at the appropriately adjusted dose, once renal function has returned to normal. Nevertheless, it would be prudent to consider an alternative to trimethoprim-sulfamethoxazole. Where trimethoprim is contraindicated, a primaquine-clindamycin combination or atovaquone can be considered for PJP. ${ }^{[11]}$

\section{Conclusions}

Trimethoprim-sulfamethoxazole is a commonly used antibiotic. It has long been reported to cause at least mild hyperkalaemia, yet there have been numerous reports of life-threatening hyperkalaemia associated with its use. Certain risk factors for hyperkalaemia have been identified, namely advanced age, higher doses of trimethoprim, concomitant use of an ACE-I/ARB, and pre-existing renal impairment. Clinicians should be cognisant of these conditions before prescribing trimethoprim-sulfamethoxazole, and should measure potassium levels in high-risk patients. Hyperkalaemia should be managed aggressively regardless of its cause. If trimethoprim-sulfamethoxazole has been implicated, it should be stopped and possibly replaced with an alternative drug.

Declaration. None.

Acknowledgements. None.

Author contributions. NdP wrote the manuscript, which was reviewed by BR. Funding. None.

Conflicts of interest. None.

1. Khanagavi J, Gupta T, Aronow WS, et al. Hyperkalemia among hospitalized patients and association between duration of hyperkalemia and outcomes. Arch Med Sci 2014;10(2):251-257. https://doi. org/10.5114/aoms.2014.42577

2. McMahon GM, Mendu ML, Gibbons FK, Christopher KB. Association between hyperkalemia at critical care initiation and mortality. Intensive Care Med 2012;38(11):1834-1842. https://doi. at critical care initiation and $\mathrm{n}$ ort
$\mathrm{org} / 10.1007 / \mathrm{s} 00134-012-2636-7$

org/10.1007/s00134-012-2636-7
3. Jain N, Kotla S, Little BB, et al. Predictors of hyperkalemia and death in patients with cardiac and renal 3. Jain N, Kotla S, Little BB, et al. Predictors of hyperkalemia and death in patients with cardiac and
disease. Am J Cardiol 2012;109(10):1510-1513. https://doi.org/10.1016/j.amjcard.2012.01.367

4. Ben Salem C, Badreddine A, Fathallah N, Slim R, Hmouda H. Drug-induced hyperkalemia. Drug Saf 2014;37(9):677-692. https://doi.org/10.1007/s40264-014-0196-1

5. Pillay-van Wyk V, Msemburi W, Laubscher R, et al. Mortality trends and differentials in South Africa from 1997 to 2012: Second National Burden of Disease Study. Lancet Glob Health 2016;4(9):e642-e653. https://doi.org/10.1016/S2214-109X(16)30113-9

6. Uthman OA, Graves PM, Saunders R, Gelband H, Richardson M, Garner P. Safety of primaquine given to people with G6PD deficiency: Systematic review of prospective studies. Malar J 2017;16(1):346, https://doi.org/10.1186/s12936-017-1989-3

7. Pazhayattil GS. Drug-induced impairment of renal function. Int J Nephrol Renovasc Dis 2014; 2014(7):457-468. https://doi.org/10.2147/IINRD.S39747

8. Higashioka $\mathrm{K}$, Niiro H, Yoshida $\mathrm{K}$, et al. Renal insufficiency in concert with renin-angiotensin8. Higashioka $\mathrm{K}$, Niiro $\mathrm{H}$, Yoshida $\mathrm{K}$, et al. Renal insufficiency in concert with renin-angiotensin-
aldosterone inhibition is a major risk factor for hyperkalemia associated with low-dose aldosterone inhibition is a major risk factor for hyperkalemia associated with low-dose
trimethoprim-sulfamethoxazole in adults. Intern Med 2016;55(5):467-471. https://doi.org/10.2169/ trimethoprim-sulfamethox

9. Perazella MA. Trimethoprim-induced hyperkalaemia: Clinical data, mechanism, prevention and management. Drug Saf 2000;22(3):227-236. https://doi.org/10.2165/00002018-200022030-00006

10. Fralick M, MacDonald EM, Gomes T, et al. Co-trimoxazole and sudden death in patients receiving inhibitors of renin-angiotensin system: Population based study. BMJ 2014;349:G6196. https://doi. org/10.1136/bmj.g6196

11. Black JR, Feinberg J, Murphy RL, et al. Clindamycin and primaquine therapy for mild-to-moderate episodes of Pneumocystis carinii pneumonia in patients with AIDS: AIDS Clinical Trials Group 044. Clin Infect Dis 1994;18(6):905-913. https://doi.org/10.1093/clinids/18.6.905

Accepted 16 July 2018 